\title{
Micronutrient Deficiency Following Esophagectomy for Cancer of the Upper Gastrointestinal Tract
}

\author{
Piers R Boshier $^{1^{*}}$, Stephanie Wakefield ${ }^{2}$, Mary Hickson ${ }^{3}$ and George B Hanna ${ }^{1}$ \\ ${ }^{1}$ Department of Surgery and Cancer, Imperial College London, UK \\ ${ }^{2}$ Department of Nutrition and Dietetics, Imperial College Healthcare NHS Trust, London, UK \\ ${ }^{3}$ Therapy Services, Imperial College Healthcare NHS Trust, London, UK
}

"Corresponding author: Boshier PR, Department of Surgery and Cancer, 10th Floor, QEQM, St Mary's Hospital London, UK, Tel: (+) 44 2078862124; Fax: (+) 44 2078866309; E-mail: prb03@ic.ac.uk

Received date: 19 October 2017; Accepted date: 27 October 2017; Published date: 03 November 2017

Copyright: ( 2017 Boshier PR, et al. This is an open-access article distributed under the terms of the Creative Commons Attribution License, which permits unrestricted use, distribution, and reproduction in any medium, provided the original author and source are credited.

\begin{abstract}
Objectives: For those patients who achieve long-term survival following esophagectomy, altered digestion and malabsorption may lead to a range of adverse gastrointestinal sequelae, including micronutrient deficiencies. The aim of the current study was to determine the prevalence of specific micronutrient deficiencies in patients following esophagectomy.
\end{abstract}

Methods: Levels of vitamin A, vitamin E, vitamin D, vitamin B1, vitamin B12, folate, ferritin, zinc and calcium were measured in a single non-fasting blood sample in patients who had undergone esophagectomy. Findings were compared to both patients who had undergone gastrectomy and an age matched Western control population.

Results: Forty-Four patients (33 male, $65.5 \pm 10.2$ yrs) a median of 26 months (IQR 12-46) following either two or three stage esophagectomy were recruited. Deficiency in one or more micronutrients was observed in $64 \%$ of patients who underwent esophagectomy. Micronutrients most commonly deficient following esophagectomy were vitamin D $(21 \%)$, vitamin B12 $(32 \%)$, ferritin $(16 \%)$ and zinc $(25 \%)$. Compared to patients who underwent gastrectomy, levels of vitamin B12 and red cell folate were significantly lower, and levels of vitamin E were significantly higher in the blood of patients following esophagetomy. Compared to a Western control population the mean levels of vitamins A, E, D and B12, ferritin and zinc were lower in patients following esophagectomy.

Conclusion: Micronutrient deficiency is common after esophagectomy affecting two thirds of all patients. Regular screening of vitamin D, vitamin B12, ferritin and Zinc levels should be considered as well as prophylactic supplementation in this patient group to prevent deficiency.

Keywords: Esophagectomy; Esophageal cancer; Nutrition; Micronutrients

\section{Introduction}

Radical surgery continues to have an important role in the potentially curative management of esophageal cancer. Improvement in the overall and disease-free survival of those patients undergoing esophagectomy [1] has focused attention towards improving the long term functional and biochemical sequelae of this surgery.

Surgical reconfiguration of the upper gastrointestinal tract can be associated with adverse intestinal symptoms including: nausea; regurgitation; dysgeusia; anorexia; food aversions; diarrhoea; dumping syndrome, and; steatorrhoea. Occurrence of these symptoms can contribute to weight loss and are detrimental to the overall nutritional status of patients [2-5]. Furthermore, use of adjunctive chemo- and radio-therapy either before or after surgery may further exacerbate these symptoms.

Patients undergoing esophagectomy may lose between 5-12\% of their body weight within the first 6 months of surgery [5]. Furthermore, weight loss of $>10 \%$ one year after surgery is reported in half of patients following esophagectomy, many of whom will fail to regain their baseline weight [5]. The majority of studies reporting nutritional deficiency in the setting of esophagectomy do so without reference to micronutrient levels. Consequently, little is known about the prevalence of micronutrient deficiency after esophagectomy.

Micronutrients are compounds required, in low concentrations, by the body to support an array of physiological functions. Micronutrients may be broadly classified as either trace elements (e.g., Iron; Zinc; Copper, and; Iodine) or vitamins (e.g., A; D; E; K, and B vitamins). Whilst Haverkort et al. determined suboptimal intake of micronutrients in $90 \%$ of patients following esophagectomy [6], this study did not correlate the observed low intake of these micronutrients to levels within patients' blood. Similarly, Okada et al. [7] and Hjelms et al. [8] reported normal vitamin B12 absorption in patients following esophagectomy, but neither study measured bloods levels of this vitamin. Wang et al. reported reduced Iron and Zinc levels in the first three months after esophagectomy with only iron levels returning to baseline after this period [9]. Recently Heneghan et al. reported that deficiency in at least one fat soluble vitamin occurred in $89.7 \%$ of patients 18 to 24 months after esophagogastric cancer resection [10]. Deficiency of Vitamin A was observed most commonly in $81.5 \%$ of patients [10]. As this study did not differentiate between patients who 
Page 2 of 5

underwent either gastrectomy or esophagectomy, the influence of the latter could not be fully determined.

The aim of the current study was to determine the prevalence of specific micronutrient deficiencies in patients following esophagectomy for cancer of the upper gastrointestinal tract. Findings were compared to both patients who had undergone gastrectomy in the same centre and a Western control population.

\section{Methodology}

Local ethics committee approval was obtained for this prospective cross-sectional study and prior to recruitment all patients provided informed written consent.

All patients with cancer of the upper gastrointestinal tract who underwent esophagectomy with gastric tube reconstruction at St Mary's Hospital between July 2003 and December 2011 and who remained free of cancer recurrence were eligible for inclusion in this study.

The following demographic information was collected at the time of recruitment: age; gender; weight and height (prior to diagnosis, prior to surgery and at recruitment); details of surgery and chemotherapy; bowel habit; alcohol and tobacco consumption, and; drug history.

Postoperative micronutrient levels were measured in a single nonfasting blood sample. The following micronutrients were independently quantified by the Department of Biochemistry of Imperial College Healthcare NHS Trust: vitamin A; vitamin E; vitamin D; vitamin B1; vitamin B12; red cell folate; iron; zinc, and; calcium.

Postoperative micronutrient levels were compared to both patients recruited during the same period who had undergone either subtotal or partial gastrectomy with Roux-en-Y reconstruction as well as a Western control population using data from The National Diet and Nutrition Survey conducted in the United Kingdom [11].

Micronutrient deficiency was defined in accordance with thresholds set by The National Diet and Nutrition Survey [11] or the analyzing laboratory. Where patients were found to be deficient in any one of these micronutrients, the patient was informed, and supplementation was commenced. Both the medical team in charge of the patient's care and the patient's general practitioner were also informed of micronutrient deficiencies.

\section{Statistical analysis}

Statistical analysis was performed using the SPSS 17 software package (SPSS Inc., Chicago, USA). Unless otherwise stated values are presented as mean \pm standard deviation (SD). The prevalence and micronutrient deficiencies in postoperative and reference populations were compared by Chi-squared test. An independent sample t-test was used for comparison of micronutrient levels between esophagectomy and gastrectomy patients. Mann-Whitney U Test was used for all other pair-wise comparisons of micronutrient levels within the postesophagectomy patient group. Statistical significance was assigned to two-tailed P-values of $<0.05$.

\section{Results}

Patient demographics and details of surgical approach are presented in Table 1.

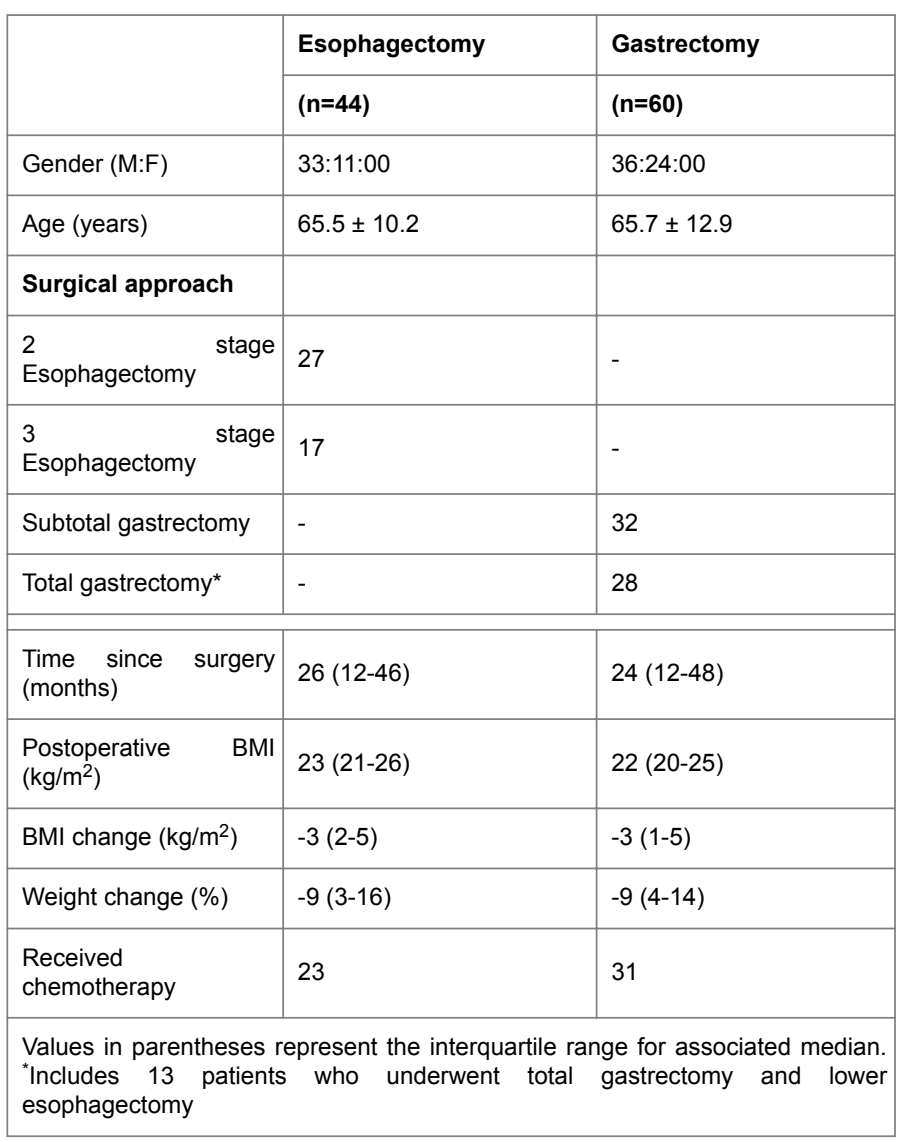

Table 1: Patient demographics and treatment characteristics.

Forty-four patients who had previously undergone esophagectomy were recruited to this study. Of this patient cohort the ratio of male to female patients was approximately 3:1 with an average age of $65.5 \pm$ 10.2 years. Recruitment occurred at a median of 26 months (IQR 12-46) after surgery at which time patients had lost a median of $9 \%$ of their preoperative body weight.

Mean micronutrient levels and rates of deficiency are presented in Tables 2 and 3 respectively. Deficiency in all micronutrients, with the exception of vitamin $\mathrm{A}$, vitamin $\mathrm{E}$ and vitamin $\mathrm{B} 1$, were observed in patients who had undergone esophagectomy. Deficiency in one or more micronutrients was observed in 29 (64\%) of patients who underwent esophagectomy. Single micronutrient deficiency was observed in $14(32 \%)$ patients. The remaining patients were deficient in either two $(n=11,25 \%)$ three $(n=1,2 \%)$ or four $(n=2,5 \%)$ micronutrients.

Of those patients who were vitamin D deficient $(n=9)$, six demonstrated evidence of secondary hyperparathyroidism, 3 of whom were calcium deficient. Ten patients with normal/low-normal vitamin $\mathrm{D}$ levels and normal calcium levels had elevated serum levels of parathyroid hormone. Of the two remaining patients found to be deficient in calcium following esophagectomy, both had vitamin D levels at the lower limit of the normal range and one had elevated serum levels of parathyroid hormone.

Median ferritin levels were lower in females compared to males (17 vs. $35 \mu \mathrm{g} / \mathrm{L} ; \mathrm{P}=0.033)$ and lower in patients who underwent three stage compared to two stage esophagectomy (18 vs. $49 \mu \mathrm{g} / \mathrm{L} ; \mathrm{P}=0.004$ ). 
Citation: Boshier PR, Wakefield S, Hickson M, Hanna GB (2017) Micronutrient Deficiency Following Esophagectomy for Cancer of the Upper Gastrointestinal Tract. Vitam Miner 6: 172.

Page 3 of 5

Absolute levels of postoperative micronutrients were not significantly altered by the administration of chemotherapy $(\mathrm{P}>0.05)$.

\section{Comparison between esophagectomy and gastrostomy patients}

Sixty patients who underwent either partial or total gastrectomy served as a positive control group. Levels of vitamin $\mathrm{B} 12(\mathrm{P}<0.001)$ and red cell folate $(\mathrm{P}=0.045)$ were significantly lower and levels of vitamin $\mathrm{E}(\mathrm{P}=0.017)$ were significantly higher in the blood of patients following esophagectomy compared to gastrectomy (Table 2). Compared to patients who underwent esophagectomy vitamin $\mathrm{D} \quad(\mathrm{P}=0.004)$ deficiency was more prevalent following gastrectomy. The prevalence of all other micronutrient deficiencies was equivalent in groups who had undergone either esophagectomy or gastrectomy.

\section{Comparison between esophagectomy patients and a Western reference population}

Compared to a Western reference population the mean levels of vitamins $\mathrm{A}, \mathrm{E}, \mathrm{D}$ and $\mathrm{B} 12$, ferritin and zinc were lower in patients following esophagectomy (comparative data from the National Diet and Nutrition Survey for vitamin B1 and calcium were however not available) (Table 2).

\begin{tabular}{|c|c|c|c|c|}
\hline & & \multirow{2}{*}{ Esophagectomy $(n=44)$} & \multirow{2}{*}{ Gastrectomy $(n=60)$} & UK population \\
\hline & & & & $\geq 65 \mathrm{yrs}(n=338)^{*}$ \\
\hline & & Mean $( \pm S D)$ & Mean $( \pm S D)$ & Mean $( \pm S D)$ \\
\hline Vitamin A & $\mathrm{mmol} / \mathrm{L}$ & $2.04( \pm 0.95)$ & $1.68( \pm 0.80)$ & $2.21( \pm 0.6)$ \\
\hline Vitamin E & $\mathrm{mmol} / \mathrm{L}$ & $29.7( \pm 7.7)$ & $25.3( \pm 8.4)^{1}$ & $33.2( \pm 8.4)$ \\
\hline Vitamin D & $\mathrm{nmol} / \mathrm{L}$ & $44( \pm 24)$ & $38( \pm 25)$ & $45( \pm 21)$ \\
\hline Vitamin B1 & $\mathrm{nmol} / \mathrm{L}$ & $155( \pm 32)$ & $148( \pm 32)$ & - \\
\hline Vitamin B12 & $\mathrm{ng} / \mathrm{L}$ & $294( \pm 161)$ & $544( \pm 499)^{2}$ & $366( \pm 142)$ \\
\hline Red Cell Folate & $\mathrm{mg} / \mathrm{L}$ & $377( \pm 219)$ & $463( \pm 251)^{3}$ & $336( \pm 167)$ \\
\hline Ferritin & $\mathrm{mg} / \mathrm{L}$ & $55( \pm 67)$ & $63( \pm 68)$ & $133( \pm 139)$ \\
\hline Zinc & $\mathrm{mmol} / \mathrm{L}$ & $11.3( \pm 1.8)$ & $10.9( \pm 2.0)$ & $14.0( \pm 3.0)$ \\
\hline Calcium & $\mathrm{mmol} / \mathrm{L}$ & $2.34( \pm 0.10)$ & $2.31( \pm 0.16)$ & - \\
\hline
\end{tabular}

Table 2: Mean micronutrient levels.

Vitamin B12 $(\mathrm{P}<0.001)$ and ferritin $(\mathrm{P}=0.016)$ deficiency was more commonly observed following esophagectomy than in a Western control population.

\section{Discussion}

This is the first study to specifically evaluate deficiencies in common micronutrients following esophagectomy for cancer of the upper gastrointestinal tract. The principal findings of this study are: (i) micronutrient deficiency is common after esophagectomy affecting $64 \%$ of all patients; (ii) high rates of vitamin B12 (32\%), zinc (25\%), vitamin D (21\%) and ferritin (16\%) deficiency following esophagectomy, and; (iii) the prevalence of micronutrient deficiency after esophagectomy is broadly equivalent to that which is observed after gastrectomy.
Micronutrients are recognized to be of importance to a range of cellular functions including epigenetic alterations that influence gene expression, cellular proliferation and differentiation [12]. Although commonly reported after gastrectomy [13-15], little is known regarding the prevalence and implications of micronutrient deficiency after esophagectomy. Whilst previous studies have focused on aspects related to micronutrient intake and absorption after esophagectomy, they do so without direct correlation to blood levels $[6-8,16]$. Wang et al. reported a persistent decrease in Zinc levels after esophagectomy [9], a finding that was corroborated by the current study. Other studies have suggested a role for micronutrients in the pathogenesis of esophageal cancer [12,17-24]. Whether changes in the levels of these micronutrients following esophagectomy influence physiological status, tumor recurrence and long-term survival is yet to be determined (Table 3 ).

\begin{tabular}{|l|l|l|l|l|}
\hline & Deficiency & Oeosphagectomy $\mathbf{( n = 4 4 )}$ & Gastrectomy $\mathbf{( n = 6 0 )}$ & UK population \\
\cline { 3 - 5 } & & & & $(\mathbf{n}=\mathbf{3 3 8})^{*}$ \\
\hline Vitamin A & $<0.70 \mathrm{mmol} / \mathrm{L}$ & $0(0.0)$ & $5(8.3)$ & 0 \\
\hline Vitamin E & $<12.0 \mathrm{mmol} / \mathrm{L}$ & $0(0.0)$ & $2(3.3)$ & $(0.0)^{* *}$ \\
\hline
\end{tabular}


Page 4 of 5

\begin{tabular}{|l|l|l|l|l|}
\hline Vitamin D & $<25 \mathrm{nmol} / \mathrm{L}$ & $9(20.5)$ & $29(48.3)^{1}$ & -21 \\
\hline Vitamin B1 & $<50 \mathrm{nmol} / \mathrm{L}$ & $0(0.0)$ & $0(0.0)$ & $(0.3)^{* * *}$ \\
\hline Vitamin B12 & $<200 \mathrm{ng} / \mathrm{L}$ & $14(31.8)$ & $14(23.3)$ & $(5.9)^{2}$ \\
\hline Red Cell Folate & $<150 \mathrm{mg} / \mathrm{L}$ & $1(2.3)$ & $1(1.7)$ & -9.3 \\
\hline Ferritin & $<15 \mathrm{mg} / \mathrm{L}$ & $7(15.9)$ & $10(16.7)$ & $(6.0)^{3}$ \\
\hline Zinc & $<10 \mathrm{mmol} / \mathrm{L}$ & $11(25.0)$ & $16(26.7)$ & - \\
\hline Calcium & $<2.25 \mathrm{mmol} / \mathrm{L}$ & $5(11.4)$ & $8(13.3)$ & - \\
\hline $\begin{array}{l}\text { Values in parentheses are percentages. }{ }^{*} \text { The National Diet and Nutrition Survey conducted in the United Kingdom [11], Average sample size 338 (range 332-349). } \\
\text { *0ficiency defined as } \alpha \text {-tocopherol to cholesterol ratio }<2.25 \mu \text { mol/mmol. }\end{array}{ }^{* * *}$ Deficiency defined as Erythrocyte transketolase activation coefficient (ETKAC) \% above \\
\hline
\end{tabular}

Table 3: Rates of micronutrient deficiency.

There are currently no agreed guidelines for the assessment and management of micronutrient deficiency after esophagectomy. Accordingly, a decision to investigate patients and initiate supplementation varies between patients and different surgical units. Due to the observed high prevalence and potential adverse effects of vitamin D, vitamin B12, Ferritin and Zinc deficiency after esophagectomy a recommendation for regular monitoring of these micronutrients and/or prophylactic supplementation may be considered. Future studies should seek to measure the treatment response to a supplementation programmed in an effort to determine the best method of overcoming and preventing these deficiencies and informing management guidelines.

Whilst biochemical markers of bone disease 25-Hydroxyvitamin D, parathyroid hormone and corrected calcium were measured during the course of this study, concurrent radiological assessment of bone mineral density was not however performed. Therefore, although one fifth of patients who underwent esophagectomy were deficient in vitamin $\mathrm{D}$, it is not possible to comment on their bone status. The finding of severely depleted vitamin D levels and elevated parathyroid hormone in several patients with frequent sun exposure and no weight loss does however strengthen the argument for a relationship between this surgery and malabsorption.

A cross-sectional design of this study was selected to ensure greater patient accrual following what is a relatively low volume surgical approach. An acknowledged limitation of this study design was the inability to draw comparison between pre- and postoperative micronutrient levels, which would in turn strengthen the proposed causal relationship between surgery and deficiencies. This limitation was partly mitigated by comparison of post esophagectomy patients to a national reference population. Esophagectomy patient were also compared to an equivalent patient population who underwent gastrectomy in the same institution who acted as positive controls. Future studies should seek to determine micronutrient levels both before and at serial intervals after surgery and may include assessment of nutrient intake and absorption. Furthermore, future studies should incorporate qualitative and quantitative assessment of nutritional status and malabsorption, similar to those utilized by Heneghan et al. [10]. Specifically, this should include characterization of postoperative dumping syndrome, steatorrhoea and bacterial overgrowth through symptom reporting and assessment of gastric empting, faecal elastase and hydrogen breath testing. Finally, studies should be adequately powered to determine the influence or patient demographics on the levels of individual micronutrients. Such studies may help to better define the mechanistic changes and risk factors for the development of micronutrient deficiencies after esophagectomy and identify those patients and most risk

\section{Conclusion}

The findings of the current study suggest a high prevalence of micronutrient deficiencies following esophagectomy, with two thirds of all patients affected. Pending further studies and establishment of accepted management guidelines, routine screening of common micronutrient levels, including, vitamin D, vitamin B12, ferritin and zinc, after esophagectomy should be considered as well as prophylactic supplementation.

\section{Acknowledgements}

The authors wish to thank Dr Melody Zhifang and Dr Joseph Eliahoo for providing statistical guidance and Dr Jonathan Vernazza for supporting sample analysis. This study was supported by funding from BAPEN and Nutricia.

\section{References}

1. Hanna GB, Boshier PR, Knaggs A, Goldin R, Sasako M (2012) Improving outcomes after gastroesophageal cancer resection: can Japanese results be reproduced in Western centers? Arch Surg 147: 738-745.

2. Jaromahum J, Fowler S (2010) Lived experiences of eating after esophagectomy: a phenomenological study. Medsurg Nurs 19: 96-100.

3. Verschuur EM, Steyerberg EW, Kuipers EJ, Essink-Bot ML, Tran KT, et al. (2006) Experiences and expectations of patients after oesophageal cancer surgery: an explorative study. Eur J Cancer Care (Engl) 15: 324-332.

4. Martin L, Lagergren J, Lindblad M, Rouvelas I, Lagergren P (2007) Malnutrition after oesophageal cancer surgery in Sweden. Br J Surg 94: 1496-1500.

5. Baker M, Halliday V, Williams RN, Bowrey DJ (2015) A systematic review of the nutritional consequences of esophagectomy. Clin Nutr 35: 987-994.

6. Haverkort EB, Binnekade JM, de Haan RJ, Busch OR, van Berge Henegouwen MI, et al. (2012) Suboptimal intake of nutrients after esophagectomy with gastric tube reconstruction. J Acad Nutr Diet 112: 1080-1087. 
Citation: Boshier PR, Wakefield S, Hickson M, Hanna GB (2017) Micronutrient Deficiency Following Esophagectomy for Cancer of the Upper Gastrointestinal Tract. Vitam Miner 6: 172.

Page 5 of 5

7. Okada N, Nishimura O, Sakurai T, Tsuchihashi S, Juhri M (1986) Gastric functions in patients with the intrathoracic stomach after esophageal surgery. Ann Surg 204: 114-121.

8. Hjelms E, Thirup P, Schou L (1999) Gastric intrinsic factor production and vitamin B12 absorption after oesophageal resection using stomach as substitute. Eur J Cardiothorac Surg 16: 273-275.

9. Wang LS, Lin HY, Chang CJ, Fahn HJ, Huang MH (1998) Effects of en bloc esophagectomy on nutritional and immune status in patients with esophageal carcinoma. J Surg Oncol 67: 90-98.

10. Heneghan HM, Zaborowski A, Fanning M, McHugh A, Doyle S, et al (2015) Prospective Study of Malabsorption and Malnutrition After Esophageal and Gastric Cancer Surgery. Ann Surg 262: 803-808.

11. Bates B, Lennox A, Prentice A, Bates H, Page P, et al. (2014) National Diet and Nutrition Survey. Results from Years 1, 2, 3 and 4 (combined) of the Rolling Programme (2008/2009 - 2011/2012). Public Health England: Public Health England.

12. Dadon Bar-El S, Reifen R (2017) Vitamin A and the Epigenome. Crit Rev Food Sci Nutr 57: 2404-2411.

13. Bragelmann R, Armbrecht U, Rosemeyer D, Schneider B, Zilly W, et al (1996) Nutrient malassimilation following total gastrectomy. Sc J Gastroenterol Suppl 218: 26-33.

14. Bradley EL, Isaacs JT, Mazo JD, Hersh T, Chey WY (1977) Pathophysiology and significance of malabsorption after Roux-en-Y reconstruction. Surgery 81: 684-691.

15. Coupaye M, Riviere P, Breuil MC, Castel B, Bogard C, et al. (2014) Comparison of nutritional status during the first year after sleeve gastrectomy and Roux-en-Y gastric bypass. Obes Surg 24: 276-283.

16. Huddy JR, Macharg FM, Lawn AM, Preston SR (2013) Exocrine pancreatic insufficiency following esophagectomy. Dis Esophagus 26: 594-597.
17. Schaafsma T, Wakefield J, Hanisch R, Bray F, Schuz J, et al. (2015) Africa's Oesophageal Cancer Corridor: Geographic Variations in Incidence Correlate with Certain Micronutrient Deficiencies. 10: e0140107.

18. Mulholland HG, Murray LJ, Anderson LA, Cantwell MM (2011) Vitamin $\mathrm{D}$, calcium and dairy intake, and risk of oesophageal adenocarcinoma and its precursor conditions. Br J Nutr 106: 732-741.

19. Abnet CC, Lai B, Qiao YL, Vogt S, Luo XM et al. (2005) Zinc concentration in esophageal biopsy specimens measured by $\mathrm{x}$-ray fluorescence and esophageal cancer risk. J Natl Cancer Inst 97: 301-306.

20. Chang SC, Goldstein BY, Mu L, Cai L, You NC, et al. (2015) Plasma folate, vitamin B12, and homocysteine and cancers of the esophagus, stomach, and liver in a Chinese population. Nutr Cancer 67: 212-223.

21. Larsson SC, Giovannucci E, Wolk A (2006) Folate intake, MTHFR polymorphisms, and risk of esophageal, gastric, and pancreatic cancer: a meta-analysis. Gastroenterology 131: 1271-1283.

22. Abnet CC, Chen Y, Chow WH, Gao YT, Helzlsouer KJ, et al. (2010) Circulating 25-hydroxyvitamin D and risk of esophageal and gastric cancer: Cohort Consortium Vitamin D Pooling Project of Rarer Cancers. Am J Epidemiol 172: 94-106.

23. Taylor PR, Li B, Dawsey SM, Li JY, Yang CS, et al. (1994) Prevention of esophageal cancer: the nutrition intervention trials in Linxian, China. Linxian Nutrition Intervention Trials Study Group. Cancer Res 54: 2029-2031.

24. O'Doherty MG, Abnet CC, Murray LJ, Woodside JV, Anderson LA, et al. (2010) Iron intake and markers of iron status and risk of Barrett's esophagus and esophageal adenocarcinoma. Cancer Causes Control 21: 2269-2279. 